\title{
Screening for Cervical Cancer by Sequential Examination of the Cervix
}

\author{
Wassan Nori Mohammed Hassan $\quad$ Roaa Mokram Hamed * Wisam.Akram \\ Department of Obstetrics and Gynecology / College of Medicine / Mustansiriyah University \\ * The National Center of Hematology / Consultant and Assistant professor in the Department of Obstetrics and \\ Gynecology \\ E-mail: Dr.wassan76@uomustansiriyah.edu.iq
}

\begin{abstract}
Background: cervical cancer carries a death sentence in the mind of every patient. Since it has a long history; it can be cured if caught early. Pap smear screening has been effective in reducing rates of cervical cancer, still, it has some draws back especially low sensitivity. The study aimed to increase the detection of Pap- tests by sequential examination of the cervix by Pap smear and colposcopy and to demonstrate the demographic criteria of those affected.

Material and method: A Comparative clinical study, conducted in the department of Obstetrics and Gynecology in AL-Yarmouk hospital /Baghdad, Iraq from 2017-2018. One hundred sexually active female fits into our inclusion and exclusion criteria were invited to participate after we explain it is aim, all participant filled a questioner sheet and were screened by Pap-smear then immediately by colposcopy exam.
\end{abstract}

Result: history of post-coital bleeding and bleeding on touch scored meaningful $P$-value $<0.05$, Papsmear examination shows $20 \%$ as abnormal study stratified into 13/20 CIN I [Cervical Intraepithelial Neoplasm], 3/20 CIN II, 2/20 CIN III, and 2/20 malignant cases. The colposcopy exam showed 32/100 abnormal cases necessitating directed histological biopsies. Examination confirms 20/32 CIN I, 7/32 CIN II, 2/32 CIN III, and 3/32 cases with cervical malignancy.

Conclusions: cervical smear had specificity and positive predictive value of $100 \%$ yet it missed 11 cases diagnosed with colposcopy one was malignant. As a result, Pap-smear alone can detect up to $18 \%$ of the pre-cancerous lesion of the cervix, this figure increases to $29 \%$ when dumbing the results of the colposcopic directed biopsy. The sensitivity of the test was $62.5 \%$ with a false-negative percentage of $37.5 \%$, having a total $88 \%$ accuracy rate and a negative predictive value of $85 \%$, this study confirms the benefits of the combined method of screening and may be used to increase papsmear sensitivity.

Key Words: CIN, cervical cancer, pap-smear, colposcopy, biopsy.

\section{Introduction}

Despite revolutionary advances in medicine as a whole and the prevention and treatment of malignancies to be exact; cervical cancer is the second most frequent cancer in women with an estimated 570000 new cases in 2018 representing $7.5 \%$ of all female cancer deaths. More than $85 \%$ of these occur in less developed regions, with $87 \%$ death rates due to an advanced stage. An unfortunate consequence of poor or badly applied screening programmers, poor education, and the empowerment of women (1). 
In $70 \%$ of the cases, evidence implicates HPV infection types (16 and 18) in the subsequent development of CIN lesions - and its progression escalates cervical cancer risk (2). Invasive carcinoma of the cervix is considered to be preventable, the long pre-invasive stage (CIN) making it amenable to screening and treatment. Thus, screening programs should be an urgent public health priority because a cure can be realistically expected. Vaccines that protect against HPV 16 and 18 are approved by WHO and have been recommended for use in multiple regions around the globe (3).Cervical cancer has shown a strong association with several risk factors, including smoking, oral contraceptive pills usage, certain nutritional deficiencies, sexual factors (4).

Cytology-based screening strategies [Pap-smear testing] was implemented as a standard screening mandatory for every woman in the western community according to guidelines globally agreed to halt its shadows. Liquid-based cytology was introduced to enhance screening performance compared to the conventional method (5). Recently a combined HPV testing with pap-smear had widened screening intervals from 3-5 years owing to the exclusion of higher-risk patients by negative testing. Sadly, in Iraq, conventional pap-smear testing is restricted to specialized centers and infeasible in low-resource settings. Moreover; a significant limitation is the absence of public awareness of these programs (6).

One of Pap smear limitations is its low sensitivity and inability to predict progression to invasive cancer in individuals with pre-cancerous lesions [CIN] thus all women with abnormal cervical cytology should have a colposcopy assessment; a binocular microscope dedicated to exam the cervix. To exclude an invasive process, identify the extent of the abnormality and it is likely to grade all women with abnormal Pap-smear (7). By adopting the concept of opportunistic screening for attendance of the outpatients' department we aim to detect the demographic criteria, presenting features, and risk factors in those presented with CIN as a primary aim. The secondary aim was to improve the sensitivity of Pap-smear with the sequential examination by colposcopy.

\section{Materials and Methods}

Study design and setting: A Comparative clinical study was performed between October 2017 and November 2018 in the department of Obstetrics and Gynecology of Al-Yarmouk hospital, after the Ethical approval of a local committee and informed consent of all participants.

Inclusion criteria: sexually active female, age range between 18-55 years and agree to undergo colposcopy.

Exclusion criteria: Pregnancy, hysterectomies women, those with a history of severe medical illness, history of invasive malignancy, women with chronic renal failure, liver failure, diabetic, blood dyscrasias all were an exclusion from the study.

Study population and Research plan: One hundred symptomatic volunteers were recruited in this study, attended the outpatient clinic for various gynecological complain in Al-Yarmouk Hospital We explain our aim and invited them to participate. A detailed history was taken and the demographic characteristics were assessed through a questionnaire sheet. 
Questionnaire Sheet

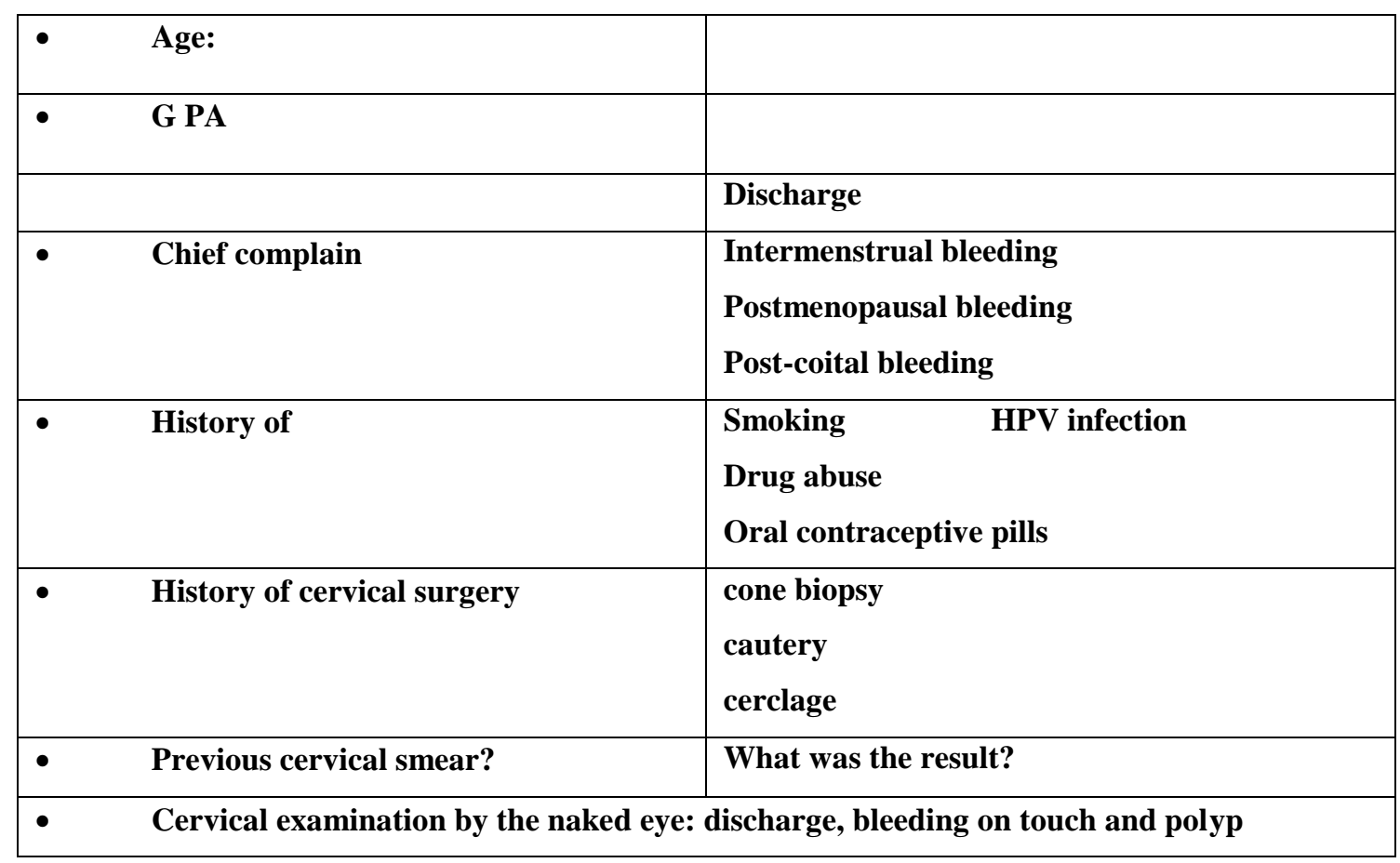

All participants were subjected to the cytological examination of the cervix immediately followed by a standard colposcopy for all patients. Each patient received a serial number and all data were stored.

Procedures for Pap-smear and colposcopy: We used the traditional Ayer's spatula to collect cervical cells exfoliated, stained according to Papanicolaou technique, and they were sent for cytological interpretation according to Bethesda reporting system and to Koss, by the same pathologist to standardize the results of smear.

A standard colposcopy was performed for all patients where at the beginning of examination the cervix was mobbed by cotton wool swab moistened with saline solution and cleaned gently. 5\% acetic acid was applied and the parameters were studied under green light: Vascular changes, degree of aceto-white area, the border characteristics, surface, pattern, and areas of lesions under study, including ulcers, polyps for their localities, number and surface character.

By highlighting precancerous lesions in a way that can be seen by the naked eye, visual inspection techniques can be administered in clinics by trained non-physician clinicians for immediate results. The ectocervix was painted by Lugol's Iodine, a colposcopy directed biopsy was taken from the non-stained areas (schiller's test positive), and immediately fixed in formalin solution and sent for histopathological study.

\section{Statistical analysis}

Data was collected and analyzed using SPSS version 10.0 for windows (SPSS, Chicago, Illinois, and the USA). Sensitivity, specificity, positive predictive value, negative predictive value, and the p-value were used 
for comparison of means.Chi-squire test which is a probability test to detect the signs of the relation between various variables. P-value $<0.05$ was considered statistically significant.

\section{Results and Discussions}

One hundred patients were enrolled in the study, their demographic characteristic was

Age range: 19-52 years; a mean was 36.0 years, parity range: 1-6, a mean was 3.37. Naked eye examination of the cervix in patients participating in the study showed discharge in 39 cases, bleeding on touch in 12 cases, polyp in 3 cases.

Pap-smear results of the patients who participated in the study were: inflammatory changes in 57/100, 23/100 normal examination and 20/100 had abnormal pap testing. For those with inflammatory changes, the non-specific infection was diagnosed in $75 \%$, specific infection including monilial in $16 \%$, and trichomonas vaginalis in $14 \%$.

Of the 20/100 abnormal testing 13/20 shows CIN I, 3/20 CIN II, 2/20 CIN III, and 2/20 malignant cases. Illustrated in Table (1a) and (1b) based on the most used histopathological classification KOSS and Bethesda system.

Table (1a): show the results of pap smear testing in patients by KOSS classification,1b in Bethesda classification

\begin{tabular}{|l|c|c|}
\hline Pap smear & No & $\%$ \\
\hline Low-grade squamous intra epithelial lesion (LSIL) & 13 & $13.0 \%$ \\
\hline High-grade squamous intra epithelial lesion (HSIL) & 5 & $5.0 \%$ \\
\hline Squamous cell carcinoma & 2 & $2.0 \%$ \\
\hline Infection & 57 & $\mathbf{5 7 . 0 \%}$ \\
\hline Normal & 23 & $\mathbf{2 3 . 0 \%}$ \\
\hline Total & 100 & $\mathbf{1 0 0 \%}$ \\
\hline
\end{tabular}

Table (1b): The pap smear (CIN) diagnosis by a cervical smear of patients, with the grading of dysplastic changes according to KOSS*

\begin{tabular}{|l|c|c|}
\hline Pap grad of SIL & No & $\%$ \\
\hline CIN I mild dysplasia & 13 & $\mathbf{6 5 . 0 \%}$ \\
\hline CIN II moderate dysplasia & 3 & $15.0 \%$ \\
\hline CIN III severe dysplasia & 2 & $\mathbf{1 0 . 0 \%}$ \\
\hline Sec & 2 & $\mathbf{1 0 . 0} \%$ \\
\hline Total No. & 20 Cases & $\mathbf{1 0 0 \%}$ \\
\hline
\end{tabular}

NB: CIN 1 comprise LSIL, CIN 2 and 3 comprise HSIL.

Patients undergo colposcopy examination of the cervix, 48/100 patients had benign findings, including inflammations, discharge, atrophic changes, and polyps. 
32/100 patients had abnormal findings for which colposcopy directed biopsies were taken to confirm the diagnoses, 20/100 of them had normal colposcopy findings. The abnormal colposcopy finding was 20/100 cases had abnormal transformation zone and 12/100 cases had suspicions lesions, including abnormal growth, unhealthy looking polyps or ulcers, three were further evaluated by colposcopy directed biopsy.

Histopathological examination confirmed the presence of CIN I in 20/32 cases (65.5\%), CIN II in 7/32 cases $(21.9 \%)$, and CIN III in 2/32 cases (6.3\%). Squamous cell carcinoma was diagnosed in 3/32 cases $(9.4 \%)$.

The significance of the association between various variables taken by the questionnaire sheet and development of CIN changes were assessed in both cytology and colposcopy and histopathological examination in both Tables (2) and (3).

Neither the age nor the parity scored a significant association to the development of CIN with an insignificant $\mathrm{p}$-value of $=0.932$ and 0.477 respectively.

Table (2): the demographic presentation of a study participant in terms of age and parity

\begin{tabular}{|c|c|c|c|c|c|}
\hline & \multicolumn{4}{|c|}{ Pap grade of SIL } & \multirow{3}{*}{ P-value } \\
\hline & \multicolumn{2}{|c|}{ CIN } & \multicolumn{2}{|c|}{ Normal } & \\
\hline & No & $\%$ & No & $\%$ & \\
\hline Age groups $($ years) $<20$ & - & - & 2 & $100.0 \%$ & \multirow{6}{*}{0.856} \\
\hline $20-24$ & 3 & $20.0 \%$ & 12 & $80.0 \%$ & \\
\hline $25-29$ & 4 & $28.6 \%$ & 10 & $71.4 \%$ & \\
\hline 30-34 & 3 & $13.0 \%$ & 20 & $87.0 \%$ & \\
\hline 35-39 & 5 & $20.8 \%$ & 19 & $79.2 \%$ & \\
\hline 40 and more & 5 & $22.7 \%$ & 17 & $77.3 \%$ & \\
\hline Parity Para 1 & - & - & 4 & $100.0 \%$ & \multirow{4}{*}{0.787} \\
\hline Para 2 & 5 & $20.0 \%$ & 20 & $80.0 \%$ & \\
\hline Para 3 & 5 & $20.8 \%$ & 19 & $79.2 \%$ & \\
\hline Para 4 and more & 10 & $21.3 \%$ & 37 & $78.7 \%$ & \\
\hline Presenting symptom Vaginal discharge & 7 & $10.8 \%$ & 58 & $89.2 \%$ & \multirow{2}{*}{$0.017 *$} \\
\hline Post-coital bleeding & 7 & $41.2 \%$ & 10 & $58.8 \%$ & \\
\hline
\end{tabular}


Table (3): Association between risk factors and developments of CIN compared by Chai sequr test

\begin{tabular}{|c|c|c|c|c|c|}
\hline \multirow{3}{*}{ Variables } & \multicolumn{4}{|c|}{ Biopsy grade of SIL } & \multirow[t]{3}{*}{ P-value } \\
\hline & \multicolumn{2}{|c|}{ CIN } & \multicolumn{2}{|c|}{ Normal } & \\
\hline & No & $\%$ & No & $\%$ & \\
\hline Age groups $($ years) $<20$ & - & - & 2 & $100.0 \%$ & 0.932 \\
\hline $20-24$ & 4 & $26.7 \%$ & 11 & $73.3 \%$ & \\
\hline $25-29$ & 5 & $35.7 \%$ & 9 & $64.3 \%$ & \\
\hline 30-34 & 8 & $34.8 \%$ & 15 & $65.2 \%$ & \\
\hline 35-39 & 8 & $33.3 \%$ & 16 & $66.7 \%$ & \\
\hline 40 and more & 7 & $31.8 \%$ & 15 & $68.2 \%$ & \\
\hline Parity Para 1 & - & - & 4 & $100.0 \%$ & 0.477 \\
\hline Para 2 & 7 & $28.0 \%$ & 18 & $72.0 \%$ & \\
\hline Para 3 & 9 & $37.5 \%$ & 15 & $62.5 \%$ & \\
\hline Para 4 and more & 16 & $34.0 \%$ & 31 & $66.0 \%$ & \\
\hline Presenting symptom Vaginal discharge & 12 & $18.5 \%$ & 53 & $81.5 \%$ & 0.001* \\
\hline Post-coital bleeding & 11 & $64.7 \%$ & 6 & $35.3 \%$ & \\
\hline Postmenopausal bleeding & 2 & $66.7 \%$ & 1 & $33.3 \%$ & \\
\hline Interemnstrual bleeding & 7 & $46.7 \%$ & 8 & $\mathbf{5 3 . 3 \%}$ & \\
\hline Smoking Not smoker & 20 & $51.3 \%$ & 19 & $48.7 \%$ & 0.004* \\
\hline Passive smoker & 8 & $19.0 \%$ & 34 & $\mathbf{8 1 . 0 \%}$ & \\
\hline Active smoker & 4 & $21.1 \%$ & 15 & $78.9 \%$ & \\
\hline Oral contraceptive pills user OCCP user & 6 & $18.8 \%$ & 26 & $\mathbf{8 1 . 3 \%}$ & 0.051 \\
\hline Not & 26 & $38.2 \%$ & 42 & $61.8 \%$ & \\
\hline IUCD user IUCDP user & 5 & $20.8 \%$ & 19 & $79.2 \%$ & 0.179 \\
\hline Not & 27 & $35.5 \%$ & 49 & $64.5 \%$ & \\
\hline HPV Positive & 4 & $40.0 \%$ & 6 & $60.0 \%$ & 0.568 \\
\hline Negative & 28 & $31.1 \%$ & 62 & $68.9 \%$ & \\
\hline
\end{tabular}

*means significant 


\begin{tabular}{|c|c|c|c|c|c|}
\hline Circulage & - & - & 5 & $100.0 \%$ & \\
\hline Cone biopsy & 1 & $100.0 \%$ & - & - & \\
\hline No & 27 & $45.0 \%$ & 33 & $55.0 \%$ & \\
\hline Discharge Yes & 19 & $48.7 \%$ & 20 & $51.3 \%$ & \multirow[t]{2}{*}{$0.004^{*}$} \\
\hline No & 13 & $21.3 \%$ & 48 & $78.7 \%$ & \\
\hline Bleeding on touch Yes & 8 & $66.7 \%$ & 4 & $33.3 \%$ & \multirow[t]{2}{*}{$0.006^{*}$} \\
\hline No & 24 & $27.3 \%$ & 64 & $72.7 \%$ & \\
\hline Polyp Yes & 1 & $33.3 \%$ & 2 & $66.7 \%$ & 0.960 \\
\hline No & 31 & $32.0 \%$ & 66 & $68.0 \%$ & \\
\hline
\end{tabular}

The same is said about smoking history, oral contraceptive pills, and using an intrauterine device with a non-meaningful p-value of $0.53,0.2$, and 0.3 respectively.

However, a history of bleeding and vaginal discharge as a presenting symptom along with easily bleeding on touch by naked eye examination scored a significant association to CIN development by a significant pvalue of $0.002,0.03$, and 0.006 respectively.

\section{Discussion}

Pap-smear is used as a routine mass screening procedure for early detection of cervical cancer in many countries however in Iraq it is limited only to refer cases still the utility of screening tests is now under question concerning effectiveness and how we can improve its productivity. Many trials have combined immediate colposcopy exams with HPV testing yet it carries high coasts and psychological distress in low rescores countries. As for immediate visualization of the cervix fallowing acetic acid application; it can be of benefit with reasonable cost-effectiveness.

Based on our data analyses we expect to have CIN in a patient who is probably in her late 30 (38years) having 4 children and more presented with a long history of vaginal discharge or maybe post-coital bleeding probably a nonsmoker with no history of oral contraceptive pills intake nor IUCD usage and had cervical surgery, those are the demographic characteristic and risk factors of our study group diagnosed with CIN.

The mean age of study participants was (35.0) versus (38) years for normal versus CIN patients, it was in line with (8) study was the mean age for patients was 38.6 years. We failed to detect a statistically significant between CIN and increasing age with a p-value $=0.932$ in contrast to the result of the (9) study showed 68 of the patients in the 5th decade and malign in 6th decade. This may be explained if we look at the age range of our patients only 7 cases were PMW mean age was young age group, so stratification of patients was inconsistent $(8,9)$. 
The mean parity of the participant enrolled was 3.5 compared to 4 for CIN patients, with non-significant $p$ value $=0.477$. Contradicting (10) who saw increased risk by increasing parity implicating childbirth for the increase in cervical carcinogenesis in addition to the risk associated with persistent HPV infection; he considered Parity as a cofactor for high-grade cervical disease among women with persistent human papillomavirus infection a factor was not taken by our study.

Regarding the presenting symptoms of the volunteer, All the participants were symptomatic having vaginal discharge in $65 \%$ compared to $(54.5 \%)$ in (8) study intermenstrual bleeding $15 \%$ in our study compared to $(19.5 \%)$, postcoital bleeding $17 \%$ in our study to (10.5\%), and postmenopausal bleeding $3 \%$ in ours to $(9 \%)$ compared to (8). It is worth mentioning that the most statistically significant symptoms concerning CIN changes were post-coital bleeding and post-menopausal bleeding with an incidence of $64.7 \%$ and $66.7 \%$ respectively and a $p$-value of $0.001(8)$.

Among the risk factor detected from the history, smoking status was associated with a high incidence of CIN, consistence with (11) study with a meaningful a $p$-value of 0.004 , Interestingly (12) disagrees suggesting it is related to the risk of infection by the HPV rather than the risk of progression for high-grade CIN no effect of smoking on CIN risk was observed.

However, this result may be due to the dilution of current smoking's effect by the inclusion of former smokers.

Intrauterine device users show no association with CIN changes with a p-value $=0.179$, (13) showed a protective effect for CIN \& cervical CA among IUCD users; he declares it plays a protective co-factor in carcinogenesis cellular immunity triggered may explain these findings.

Surprisingly; a history of HPV infection did not show significant association with CIN changes in our study having a p-value of 0.56 such history was confirmed by the site of the warty lesions and modalities of treatment, it is worth saying that history of Human Papillomavirus infection was detected in only 10 of our patients, including 4 diagnosed with changes. This can explain HPV is considered a stigma not acknowledged by most of our patients.

Human Papillomavirus infection is a major prerequisite for cervical carcinogenesis thus, the true figure may be higher, this contradicts most of the studies in this matter as (14).

The history of cervical surgery was positive regarding cautery with a significant p-value of 0.001 . This may be a bias study due to miss belief by most of our patients that cauterization cures everything!

Pap smear testing according to the Bethesda reporting system shows $20 \%$ abnormal reports as a low-grade squamous intraepithelial lesion LSIL, high-grade squamous intraepithelial lesions, and squamous cell carcinoma in $13 \%, 3 \%$, and $2 \%$ respectively compared to Shashwat Vidyadha. The study, ASC-US was found in 5.8\% of the women and ASC-H was found in 1.4\% of the women. LSIL was found in $6.7 \%$ of the women, and HSIL was found in $6.2 \%$ of the women. Normal findings were seen in (79.3\%) compared to $57 \%$ in our study (15).

Upon colposcopic examination, the abnormal examination was seen in 32/100 patients, as abnormality in the transformation zone and suspicious lesions, including abnormal growth, unhealthy looking polyps, or 
ulcers evaluated by colposcopy directed. Compared to (16) where $(15.9 \%)$ of cases show an unhealthy cervix which bleeds easily on touch; clinical diagnosis of carcinoma of cervix comprised (12.8\%) of their cases compared to $(9.4 \%)$ in our analyses. Higher detection rates in their analyses can be due to a higher sample size and wider inclusion criteria (16).

CIN changes diagnosed by cervical smear alone were 18 cases (18\%) versus $9 \%$ in (8). This figure increased to 29 cases (29\%) when combining the results of colposcopy and biopsy compared to 31 cases in $(8,16)$.

Pap -smear failed to diagnose malignant changes in one patient where the report showed HSIL plus CIN III but no malignant cells, compared to the colposcopic examination which showed an abnormal transformation zone where biopsy confirmed the diagnoses of squamous cell carcinoma diagnosis of SCC was2\% in our study compared to $6.2 \%$ in (16), Besides pap-smear failed to diagnose CIN changes in 11 patients one of which shown to be squamous cell carcinoma, compared to 9 missed cases in (16) this may be due to poor interpretation of the cytological examination of our labs or maybe an error in technique.

The sensitivity of pap-smear was $62.5 \%$ with a false-negative percentage of $37.5 \%$, having a total $88 \%$ accuracy rate \& negative predictive value of $85 \%$. In Shashwat Vidyadha.

The sensitivity of PAP smear was $29.7 \%$, specificity was $94.4 \%$, PPV was $70.4 \%$, NPV was $75.1 \%$ and accuracy was $74.5 \%$ for diagnosing premalignant lesions of the cervix(15).

The diagnosis of CIN was confirmed by colposcopic \& histopathological examination in all patients, showed CINI changes in (20\%) opposed to $32 \%$, CIN II in $7 \%$ compared to (17\%) \& CIN III in (3\%) compared to $5 \%$ in (16).

What is a novel about this study that sets the most important risk factors for Iraqi females both by history and clinical exam; moreover it has integrated those risk factors along with pap smear testing and colposcopic exam in one shell improving its detection rates.

Limitation of this study includes, we have adapted conventional not the liquid-based cytology which tends to collect the whole sample from the sampling devise in a liquid media \& send for laboratory evaluation, This method is recommended to become the standard test, but it is more expensive(5). We did not use testing for HPV by serology; but by history; which is the gold standard along with Pap-smear testing, being an expensive test that cannot be afforded by all.

Points of the strength of this study are adding the naked eye examination to the cervix will improve the detection rate by Pap-smear without adding extra charge nor burden to both medical staff and patients; the best coast benefit. This approach has already been adopted in areas with low resources setting in Africa adding the benefit of immediate diagnosis and treatment; see and treat strategy. Still, a major side effect is overdiagnosis, and the absence of pathological samples for histology if the ablative approach rather than an excisional approach is used. It is worth saying both methods have similar success rates in treating CINI, II (17).

A good trained medical staff in the examination technique, increasing the public aware of the importance of Pap -smear testing through health institutions and local community agencies, will empower screening programs 


\section{Conclusions}

Iraqi women caries a CIN diagnosis can be expected to be her late 30, multiparous women with a presenting symptom of vaginal discharge or post-coital bleeding. Mostly she is a non-smoker with no history of oral contraceptive pills intake nor IUCD usage and had cervical surgery. The sensitivity of Pap-smear can be increased if we add a colposcopy exam; besides the naked eye exam by acetic acid along with selected risk factors all integrated into one prediction module. Detection rates both for CIN changes and malignant changes will empower Iraqi women to thrive crowned with health via early screening programs at the bestafforded cost.

\section{References}

1. Du T, George C. The contextual quality of life of women with cervical cancer in the Western Cape, South Africa. Diss. Stellenbosch: Stellenbosch University. (2018).

2. Garland SM, Paavonen J, Jaisamrarn U, Naud P, Salmerón J, Chow SN, Apter D, Castellsagué X, Teixeira JC, Skinner SR, Hedrick J. Prior human papillomavirus-16/18 AS04-adjuvanted vaccination prevents recurrent high grade cervical intraepithelial neoplasia after definitive surgical therapy: Post-hoc analysis from a randomized controlled trial. International journal of cancer. (2016) Dec 15; 139(12): 28122826.

3. Hancock G, Hellner K, Dorrell L. Therapeutic HPV vaccines. Best Practice and Research Clinical Obstetrics and Gynaecology. (2018) Feb 1; 47: 59-72.

4. Akram Husain RS, Ramakrishnan V. A review of risk factors in the development of cervical malignancy. Cancer Sci Res Open Access. (2016); 3(1): 1-4.

5. Lim SC, Yoo CW. Current status and perspectives on cervical cancer screening in Korea. Journal of Pathology and Translational Medicine. (2019) Jul; 53(4): 210.

6. Lewitowicz P, Nasierowska-Guttmejer A, Rokita W, Adamczyk-Gruszka O, Gluszek S, Chrapek M, Kolos M, Wrona-Cyranowska A, Misiek M. HPV genotyping and p16/Ki-67 test significantly improve detection rate of high-grade cervical squamous intraepithelial lesion. Archives of Medical Science: AMS. (2020); 16(1): 87.

7. Mustafa RA, Santesso N, Khatib R, Mustafa AA, Wiercioch W, Kehar R, Gandhi S, Chen Y, Cheung A, Hopkins J, Ma B. Systematic reviews and meta-analyses of the accuracy of HPV tests, visual inspection with acetic acid, cytology, and colposcopy. International Journal of Gynecology and Obstetrics. (2016) Mar 1; 132(3): 259-265.

8. Singhal A, Raina RK, Verma S, Verma A. Predictive accuracy of cervical cytology and colposcopy in diagnosing premalignant and malignant cervical lesions: A hospital-based study from the sub-Himalayan region of Indian subcontinent. CHRISMED Journal of Health and Research. (2019) Jan 1; 6 (1): 39.

9. Bariha Kalpana, S. Madanan A, Baniwal D - Pap Smear Versus Colposcopy for Diagnosis of Precancerous Lesions of Cervix International Journal of scientific research and management Published (2016). 
10. Jensen KE, Schmiedel S, Norrild B, Frederiksen K, I ftner T, Kjaer SK. Parity as a cofactor for highgrade cervical disease among women with persistent human papillomavirus infection: a 13-year follow-up. British journal of cancer. (2013) Jan; 108(1): 234-239.

11. Min KJ, Lee JK, So KA, Kim MK. Association between passive smoking and the risk of cervical intraepithelial neoplasia 1 in Korean women. Journal of Epidemiology. (2017) :JE20160118.

12. Syrjänen, Kari, et al. Smoking is an independent risk factor for oncogenic human papillomavirus (HPV) infections but not for high-grade CIN. European journal of epidemiology. (2007): 22(10): 723-735.

13. Castellsagué X, Díaz M, Vaccarella S, de Sanjosé S, Muñoz N, Herrero R, Franceschi S, Meijer CJ, Bosch FX. Intrauterine device use, cervical infection with human papillomavirus, and risk of cervical cancer: a pooled analysis of 26 epidemiological studies. The lancet oncology. (2011) Oct 1; 12(11): 10231031.

14. Schiffman M, Kinney WK, Cheung LC, Gage JC, Fetterman B, Poitras NE, Lorey TS, Wentzensen N, Befano B, Schussler J, Katki HA. The relative performance of HPV and cytology components of on testing in cervical screening. JNCI: Journal of the National Cancer Institute. (2018) May 1; 110(5): 501-508.

15. Vidyadhar DS, Bhattacharya DA, Bohara DS, Dwivedi DA, Agarwal DA, Gangwar DD. Comparison and correlation of cytology, colposcopy, and histopathology of premalignant lesions of cervix in rural women of Barabanki District. IOSR J Dent Med Sci. (2017) Apr; 16(04): 13-18.

16. Makaju R, Dhakal B, Dhakal R. Clinicopathological correlation of uterine cervical lesions with focus on premalignant and malignant lesions. Journal of Pathology of Nepal. (2019) Sep 29; 9(2): 1560-1563.

17. Saslow D, Andrews KS, Manassaram-Baptiste D, Smith RA, Fontham ET. American Cancer Society Guideline Development Group. Human papillomavirus vaccination 2020 guideline update: American Cancer Society guideline adaptation. CA: A Cancer Journal for Clinicians. (2020) Jul; 70(4): 274-280.

18. Ibrahim A, Aro AR, Rasch V, Pukkala E. Cervical cancer screening in a primary health care setting .17 in Sudan: a comparative study of visual inspection with acetic acid and Pap smear. International Journal of Women's Health. (2012) : $4 ; 67$. 